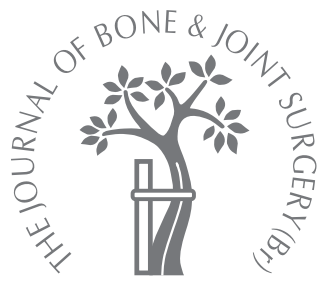

K.-Y. Ha, J.-S. Lee, K.-W. Kim, J.-S. Chon

From The Catholic University and Dongshin General Hospital, Seoul, Korea

\title{
Percutaneous vertebroplasty for vertebral compression fractures with and without intravertebral clefts
}

We present the clinical and radiological results of percutaneous vertebroplasty in the treatment of $\mathbf{5 8}$ vertebral compression fractures in $\mathbf{5 1}$ patients at a minimum follow-up of two years. Group 1 consisted of 39 patients, in whom there was no associated intravertebral cleft, whilst group 2 comprised 12 patients with an intravertebral cleft. The Oswestry disability index (ODI) and visual analogue scale (VAS) scores were recorded prospectively. The radiological evidence of kyphotic deformity, vertebral height, leakage of cement and bone resorption around the cement were studied restrospectively, both before and after operation and at the final follow-up.

The ODI and VAS scores in both groups decreased after treatment, but the mean score in group 2 was higher than that in group 1 ( $p=0.02$ (ODI), $p=0.02$ (VAS)). There was a greater initial correction of the kyphosis in group 2 than in group 1, although the difference was not statistically significant. However, loss of correction was greater in group 2. Leakage of cement was seen in 24 (41.4\%) of 58 vertebrae (group 1, 32.6\% (15 of 46); group 2, 75\% (9 of 12)), mainly of type $B$ through the basal vertebral vein in group 1 and of type $C$ through the cortical defect in group 2. Resorption of bone around the cement was seen in three vertebrae in group 2 and in one in group 1 . There were seven adjacent vertebral fractures in group 1 and one in group 2.

Percutaneous vertebroplasty is an effective treatment for osteoporotic compression fractures with or without an intravertebral cleft. Nonetheless, higher rates of complications related to the cement must be recognised in patients in the presence of an intravertebral cleft.

Percutaneous vertebroplasty was introduced by Galibert et $\mathrm{al}^{1}$ and has been used to treat patients with osteoporotic vertebral compression fractures, vertebral metastatic cancer, myeloma and haemangioma, all of which cause severe pain. A success rate of $90 \%$ to $95 \%$ has been claimed for managing osteoporotic vertebral compression fractures with this treatment. ${ }^{2,3}$ Percutaneous vertebroplasty has also been used in Kummell's disease, in which an intravertebral cleft occurs in the presence of an osteoporotic compression fracture. Jang, Kim and $\mathrm{Lee}^{4}$ and Lane et $\mathrm{al}^{5}$ reported excellent results in this situation. The intravertebral clefts, first described by Maldague, Noel and Malghem, ${ }^{6}$ are considered to represent avascular necrosis of the vertebral body and are not thought to be associated with acute osteoporotic compression fractures. ${ }^{7}$ Kummell's disease causes rapid collapse of the vertebra, loss of lordosis due to necrosis of the anterior vertebral body and fibrosis around the bone necrosis. ${ }^{7}$ As a result, there is a large void in the anterosuperior portion of the vertebral body. Lane et $\mathrm{al}^{5}$ and McKiernan and Faciszewski ${ }^{8}$ found that the filling patterns of polymethylmethacrylate (PMMA) in percutaneous vertebroplasty were different in cleft vertebrae and uncleft vertebrae.

We have compared the clinical and radiological results of percutaneous vertebroplasty in patients with osteoporotic vertebral compression fractures with or without an intravertebral cleft.

\section{Patients and Methods}

Between July 2000 and October 2002 we treated 54 patients with persistent pain due to osteoporotic vertebral compression fractures by percutaneous vertebroplasty. Two were lost to follow-up and one died, leaving 51 consecutive patients with 58 fractures who were followed up for more than two years. They were separated into two groups. Group 1 consisted of 39 patients with 46 involved vertebrae, without an associated intravertebral cleft and 


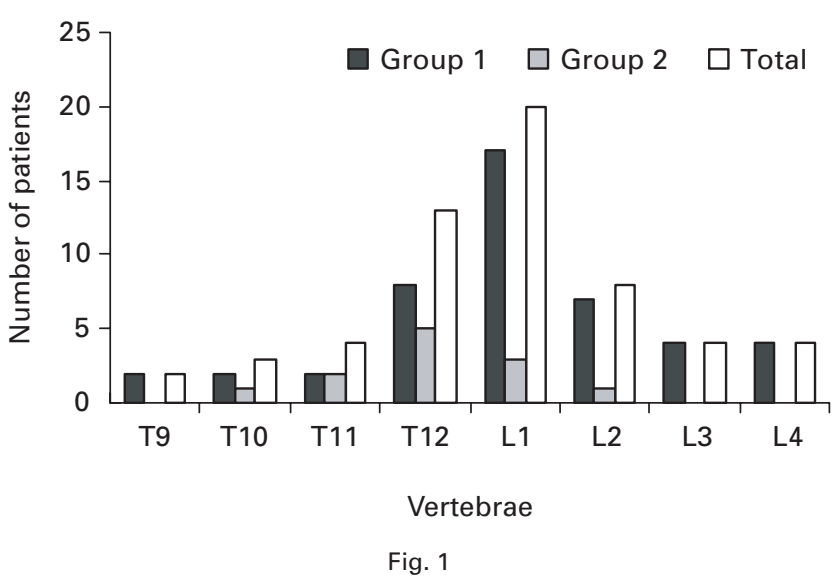

Bar chart showing the level of vertebroplasty.

group 2 comprised 12 patients with 12 vertebral compression fractures, with an intravertebral cleft. The male-tofemale ratio in group 1 and group 2 was $6: 33$ and 1:11, respectively. The mean age for all patients at the time of treatment was 70.9 years (64 to 79), with a mean age of 71.1 years for group 1 and 62.5 years for group 2 .

Of the 46 vertebral compression fractures in group 1, 32 were at T12 to L2, compared with nine at the same sites in group 2 (Fig. 1). The fractures selected for percutaneous vertebroplasty were determined from plain radiographs, bone scans, MRI and physical examination for the location of pain in patients who had no improvement in pain after three weeks of conservative treatment. From the bone scan, fracture segments showing increased uptake of isotope were selected. All patients underwent MRI and the vertebrae with intravertebral fluid or air shadows in the sagittal plane were selected and their location matched to the site of pain, in order to determine which segment should be treated. If severe vertebral compression fractures seen on plain radiographs did not show increased uptake on the bone scan, they were thought to represent old injuries.

Percutaneous vertebroplasty was undertaken under local anaesthesia, with the spine extended as described by Jensen et al. $^{3} \mathrm{~A}$ bone-biopsy needle was inserted into the anterior third of the vertebra, through a transpedicular approach, to inject the liquid bone cement under radiological control using an image intensifier. Injection continued until the vertebra was opacified, or leakage into the paraspinal veins was seen. After finishing the injection, the same procedure was repeated through the opposite pedicle. PMMA cement (Depuy International Ltd, Leeds, United Kingdom) mixed with barium sulphate contrast was used in all patients. The mean quantity of bone cement in groups 1 and 2 was $6.2 \mathrm{ml}$ and $5.4 \mathrm{ml}$, respectively. Using dual-energy x-ray absorptiometry (Hologic QDR-4500A, Bedford, Massachusetts), the bone mineral density (BMD) of the lumbar spine and proximal femur was checked in all patients who underwent percutaneous vertebroplasty. The T-score for the BMD was

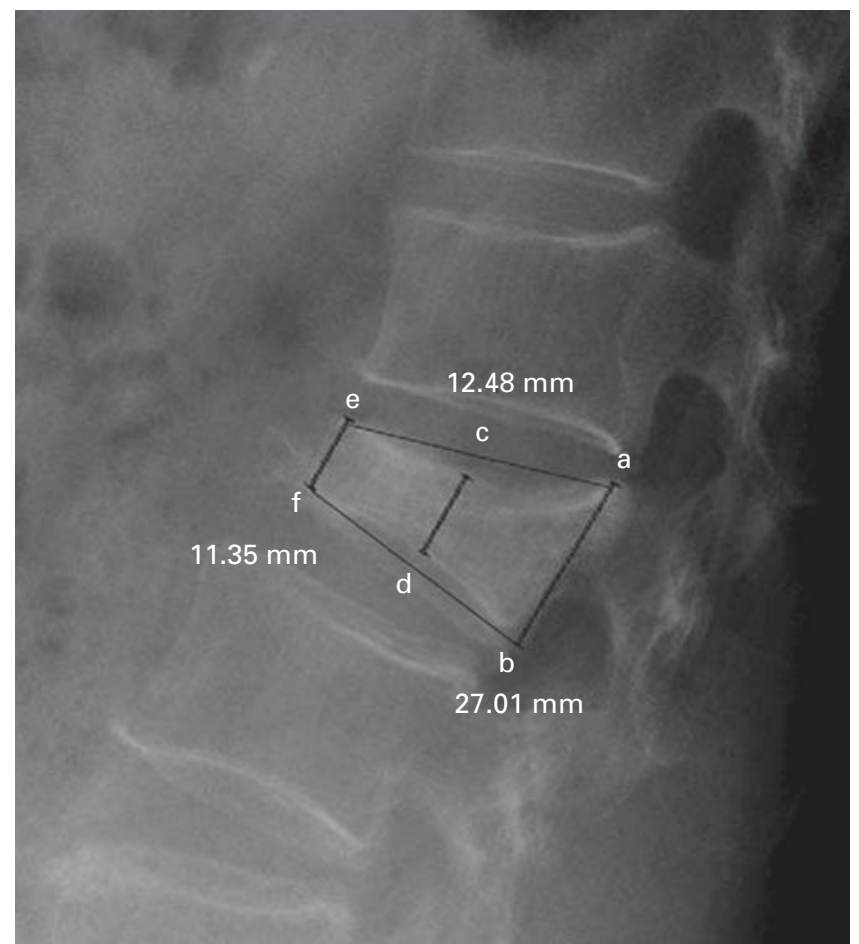

Fig. 2

Radiograph showing the measurement percentage height ratio of the vertebral height. Points (a) and (e) were placed at the most posterosuperior and anterosuperior end-plate margins, respectively and points (b) and ( $f$ ) at the most posteroinferior and anteroinferior margins. The two remaining points (c) and (d) were placed at the intersection of a perpendicular drawn from the mid-point of lines ae and bf, and the superior and inferior vertebral end-plates. Vertebral height was the distance between identical points on the superior and inferior end-plates at the posterior (line ab), middle (line cd) and anterior location (line ef). The anterior-to-posterior ratio $=\mathrm{ef} / \mathrm{ab} \times 100$, and the middle-to-posterior ratio $=\mathrm{cd} / \mathrm{ab} \times 100$.

less than -2.5 in all patients, confirming the diagnosis of osteoporosis. The mean follow-up was for 26.3 months (24 to 36 ).

In all patients, plain radiographs were obtained on the day immediately before the percutaneous vertebroplasty, one day after treatment and at the final follow-up. CT was also performed one to three days after treatment. The kyphotic angle, the vertebral height, resorption of bone around the injected cement and fractures of the adjacent vertebrae were assessed from the plain films (Fig. 2). The vertebral height was measured using the method of McKiernan, Faciszewski and Jensen. ${ }^{9}$ For pre- and postoperative comparison and for the final follow-up, we calculated the ratio of the height of the anterior and posterior aspects of the vertebral body and that of the middle and the posterior vertebral height.

Based on the post-operative CT scans, leakage of any cement was classified into three types: type B (leakage through the basal vertebral vein), type $S$ (leakage through the segmental vein) and type $\mathrm{C}$ (leakage through the site of cortical bone loss). ${ }^{10,11} \mathrm{~A}$ visual analogue scale for pain (VAS) and the Oswestry Disability Index (ODI) ${ }^{12}$ were 


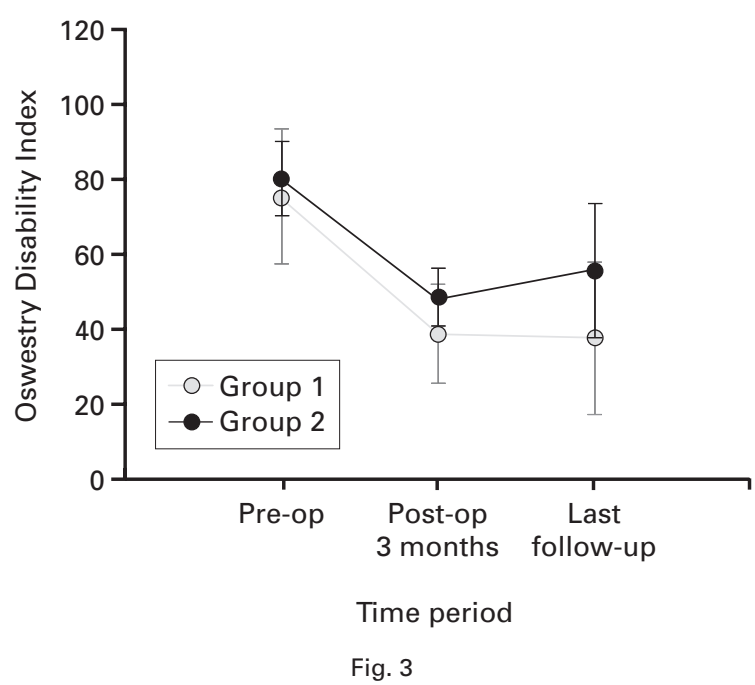

Graph showing the Oswestry disability index for vertebral compression fractures with and without an intravertebral cleft.

applied pre-operatively, three months after treatment and at the final follow-up, to compare the severity of pain and its influence on daily life.

Statistical analysis. The paired $t$-test, Fisher's exact test and the Pearson chi-squared test were used for statistical analysis. The statistical significance before and after the operation and at the final follow-up was tested by repeatedmeasures analysis of variance (ANOVA). The results were considered to be significant if $\mathrm{p}<0.05$.

\section{Results}

Clinical findings. In group 1 , the mean pre-operative ODI score of 75.1 had decreased to 38.7 by three months after the operation. The difference was statistically significant (repeated-measures ANOVA, $\mathrm{p}<0.0001$ ). The mean score at the final follow-up was 37.3 , but this further change was not statistically significant.

In group 2, the mean pre-operative ODI score of 79.9 had decreased to 48.5 by three months after the operation. The difference was statistically significant (repeated-measures ANOVA, $p<0.0001$ ). The mean score at the final follow-up had increased to 55.4, but this difference was not statistically significant (Fig. 3).

In group 1, the mean pre-operative VAS score was 8.3 and had decreased to 4.2 after the operation. The difference was statistically significant (repeated-measures ANOVA, $\mathrm{p}<0.0001$ ). The mean score at the final follow-up was 3.5, but this change was not statistically significant.

In group 2, the mean pre-operative VAS score was 9.4, which decreased to 4.5 after the operation. The difference was statistically significant (repeated-measures ANOVA, $p<0.001$ ). The mean score at the final follow-up was 5.9, which was not statistically significant from the result at three months (Fig. 4).

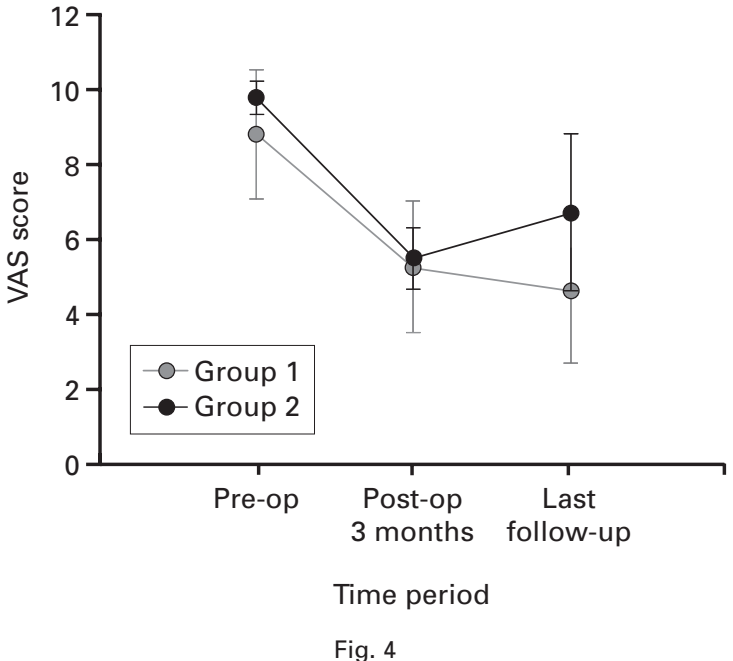

Graph showing the visual analogue scale (VAS) score for vertebral compression fractures with and without an intravertebral cleft.

At the $5 \%$ level of significance, the ODI and VAS of group 2 were significantly higher than those of group 1 (repeated-measures ANOVA, $\mathrm{p}=0.02$ ).

Radiological findings. In group 1, the mean kyphosis measured $12.0^{\circ}$ (SD 5.8) pre-operatively, $11.2^{\circ}$ (SD 5.5) postoperatively, and $11.2^{\circ}$ (SD 5.6) at the final follow-up, representing a correction of $6.7 \%$. Group 2 had a mean kyphosis of $15.5^{\circ}$ (SD 6.9) pre-operatively, $12.0^{\circ}$ (SD 4.0) post-operatively, and $12.7^{\circ}$ (SD 4.9) at the final follow-up. A correction of $18.1 \%$ was achieved, but this was not fully maintained. The difference between the two groups was not statistically significant.

In group 1 , the mean pre-operative anterior and posterior vertebral height ratio measured $70 \%$ (SD 1.5), the postoperative ratio was $72 \%$ (SD 2.2) and the ratio was $71.6 \%$ (SD 3.7) at the final follow-up. By contrast, in group 2, the mean pre-operative ratio was $60 \%$ (SD 3.2), the post-operative ratio $66 \%$ (SD 4.8 ) and at final follow-up, was $64.4 \%$ (SD 4.7). Group 2 had a larger increase in anterior height than group 1, although the difference was not statistically significant.

In group 1, the mean pre-operative middle and posterior vertebral height ratio measured $60 \%$ (SD 3.8). Post-operatively it was $68 \%$ (SD 2.6) and at final follow-up, 67.6\% (SD 1.4). This compared with group 2 , which had a mean preoperative ratio of $55 \%$ (SD 3.1), a post-operative ratio of $59 \%$ (SD 5.4) and a ratio of 58\% (SD 3.5) at final follow-up. Group 2 showed a larger increase in middle height than group 1, although the difference was not statistically significant.

Complications. Those complications directly related to the percutaneous vertebroplasty occurred in 31 of 58 vertebrae $(53.4 \%)$, but there were no neurological symptoms caused by the treatment and no infections. The Pearson chi- 


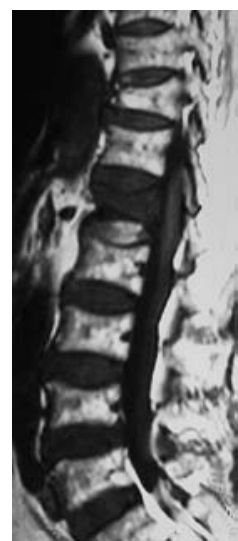

Fig. $5 a$

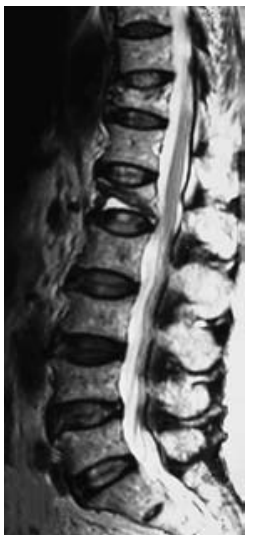

Fig. $5 b$

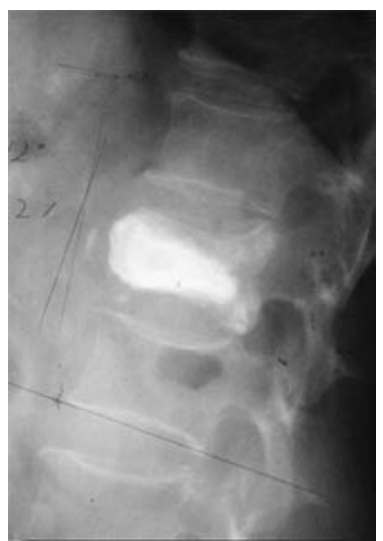

Fig. 5c

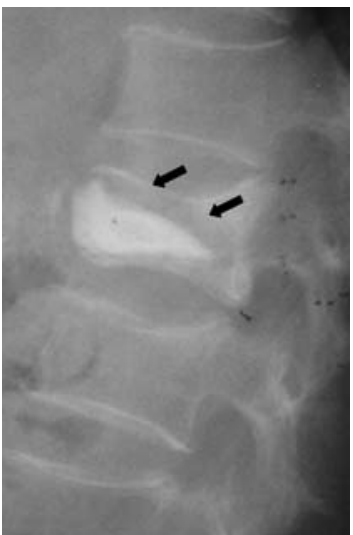

Fig. $5 d$

Figures $5 \mathrm{a}$ and $5 \mathrm{~b}-\mathrm{MRI}$ of a 78-year-old woman with a compression fracture at L1 showing a) a sagittal T1weighted scan with a high-signal cleft-like collection of fluid and b) a sagittal T2-weighted scan with a low-signal cleft. Figures $5 c$ and $5 d$ - Lateral radiographs showing $c$ ) a uniform opacity and sharp margins after vertebroplasty and d) at two months after operation there is bone resorption around the cement (arrows).

squared test was used for the statistical analysis of complications.

Leakage of bone cement was observed in 24 of 58 vertebrae $(41.4 \%)$. In group 1, leakage was seen in 15 of 46 vertebrae $(32.6 \%)$ and in 13 of these was classified as type B. In group 2, leakage was seen in nine of the 12 vertebrae $(75 \%)$ and seven were classified as type C. More leakage was observed in group 2 than in group $1(\mathrm{p}<0.05)$.

Bone resorption around the cement was observed in one of 46 vertebrae $(2.2 \%)$ in group 1 and three of $12(25 \%)$ in group 2 (Fig. 5). This difference was significant $(\mathrm{p}=0.023)$.

Fractures adjacent to the lesion occurred in five of the 51 patients $(9.8 \%)$. There were seven in four patients in group 1 and one in group 2. There was no statistical significance between the groups.

\section{Discussion}

Studies on vertebroplasty ${ }^{13-15}$ have focused mainly on its advantages, the method of operation or the results of its application to osteoporotic compression fractures. Our study compared the results of percutaneous vertebroplasty in vertebral compression fractures with or without intravertebral clefts at a minimum follow-up of two years.

The ODI and VAS scores decreased in both groups after treatment. However, group 2 showed higher scores than group 1, suggesting that patients with an intravertebral cleft experienced less reduction of pain and more inconvenience in daily life after the operation than those without a cleft. Nevertheless, percutaneous vertebroplasty provided satisfactory results in these patients. The ODI and VAS scores at the final follow-up, when compared with those immediately after operation, showed a slight decrease in patients with intravertebral clefts, whereas they increased slightly in those without a cleft (group 1). However, the difference between patients with and without a cleft was not statistically significant. This suggested that the treatment provided a comparatively well-maintained improvement.

In group 2, four of nine vertebrae with leakage of cement, three with bone resorption around the injected cement and one with a fracture in the adjacent vertebra showed an increase in the ODI and VAS scores at the final follow-up compared with those after three months. Similarly, in group 1, ten of 15 vertebrae with leakage of cement, one with bone resorption around the injected cement and seven with fractures at the adjacent vertebrae showed an increase in the ODI and VAS scores at the final follow-up, compared with those after three months.

Our patients were representative of those with vertebral compression fractures in general, with most involved vertebrae being located at the thoracolumbar junction. , $^{76,17}$

The extent of the correction of the kyphosis in our series was consistent with that of Michael et al. ${ }^{18}$ We found that the correction was partially lost at the final follow-up, in particular in patients with an intravertebral cleft. This agrees with the results of McKiernan, Jensen and Faciszewski ${ }^{19}$ who reported that posture had a greater influence on the extent of the kyphosis in patients with a cleft than in those without. McKiernan et a ${ }^{19}$ compared the pre-operative plain radiographs taken in the upright position with those taken prone, noting that the correction of the kyphotic deformity seemed to be more significant in the latter position. However, in our study all the radiographs were obtained with the patients standing. Of note was the finding that the ratio of vertebral height increased in both groups after operation but had decreased by the final follow-up.

Leakage of bone cement from the vertebra on to the dura mater has been reported in $30 \%$ to $70 \%$ of percutaneous vertebroplasties. ${ }^{20,21}$ In our study, more leakage occurred in 
the presence of a cleft and was consistent with the findings of Yeom et al, ${ }^{10,11}$ who found that in the absence of a cleft, the leakage of bone cement was mainly of type B. However, they reported that type B leakage made up about $40 \%$ of the total, which was much lower than our incidence of $86.7 \%$.

Bone resorption around the injected bone cement was found more often in vertebrae with a cleft than in those without. McKiernan and Faciszewski ${ }^{8}$ reported the patterns of opacification after percutaneous vertebroplasty and found that persistently mobile, clefted vertebrae filled as a confluent reservoir for cement, with uniform opacity and sharp radiological margins. In the absence of a cleft, the cement was interspersed throughout the trabecular space in a more even manner. We found that in vertebral compression fractures with an intravertebral cleft, the cement filled a large gap in the anterosuperior region of the vertebral body. Therefore, the load on the vertebrae was thought to be transferred to the bone cement, which was harder than the surrounding bone, thus creating a stressshielding effect. This possibly caused more bone resorption in patients with a cleft than in those without.

Our results indicate that percutaneous vertebroplasty can be used to treat osteoporotic vertebral compression fractures with or without an intravertebral cleft. However, there is a greater likelihood of complications if an intravertebral cleft is present.

We wish to thank all staff at the spine meeting of Catholic Medical Center for their suggestions in the preparation of this report.

No benefits in any form have been received or will be received from a com mercial party related directly or indirectly to the subject of this article.

\section{References}

1. Galibert P, Deramond H, Rosat P, Le Gars D. Preliminary note on the treatment of vertebral angioma by percutaneous acrylic vertebroplasty. Neurochirurgie 1987;33: $166-8$ (in French).
2. Barr JD, Barr MS, Lemley TJ, McCann RM. Percutaneous vertebroplasty for pain relief and spinal stabilization. Spine 2000;15:923-8.

3. Jensen ME, Evans AJ, Mathis JM, et al. Percutaneous polymethylmethacrylate vertebroplasty in the treatment of osteoporotic vertebral body compression fractures: technical aspects. Am J Neuroradiol 1997;18:1897-904.

4. Jang JS, Kim DY, Lee SH. Efficacy of percutaneous vertebroplasty in the treatment of intravertebral pseudarthrosis associated with noninfected avascular necrosis of the vertebral body. Spine 2003:28:1588-92.

5. Lane JI, Maus TP, Wald JT, et al. Intravertebral clefts opacified during vertebroplasty: pathogenesis, technical implications, and prognostic significance. AJNR Am J Neuroradiol 2002;23:1642-6

6. Maldague BE, Noel HM, Malghem JJ. The intravertebral vacuum cleft: a sign of ischemic vertebral collapse. Radiology 1978;129:23-9.

7. Theodorou DJ. The intravertebral vacuum cleft sign. Radiology 2001;221:787-8.

8. McKiernan F, Faciszewski T. Intravertebral clefts in osteoporotic vertebral compression fractures. Arthritis Rheum 2003;48:1414-19.

9. McKiernan F, Faciszewski T, Jensen R. Reporting height restoration in vertebral compression fractures. Spine 2003;28:2517-21.

10. Yeom JS, Kim WJ, Choy WS, et al. Bone cement leakage in vertebroplasty for osteoporotic compression fractures. J Kor Ortho Asso 2003;38:293-300.

11. Yeom JS, Kim WJ, Choy WS, et al. Leakage in cement in percutaneous transpedicular vertebroplasty for painful osteoporotic compression fractures. J Bone Joint Surg [Br] 2003;85-B:83-9.

12. Fairbank JC, Pynsent PB. The Oswestry disability index. Spine 2000;15:2940-52.

13. Cortet B, Cotton A, Boutry N, et al. Percutaneous vertebroplasty in the treatment of osteoporotic vertebral compression fractures. J Rheumatol 1999;26:2222-8.

14. Cyteval C, Sarrabere MP, Roux J0, et al. Acute osteoporotic vertebral collapse: open study on percutaneous injection of acrylic surgical cement in 20 patients. AJR Am J Roentgeno/ 1999;173:1685-90.

15. Martin JB, Jean B, Sugiu K, et al. Vertebroplasty: clinical experience and followup results. Bone 1999;25(2 Suppl):11-15.

16. Ismail AA, Cooper C, Felsenberg D, et al. Number and type of vertebral deformities: epidemiological characteristics and relation to back pain and height loss. Osteoporos Int 1999;9:206-13.

17. Kumpan W, Salomonowiz E, SeidI G, Wittich GR. The intravertebral vacuum phenomenon. Skeletal Radiol 1986;15:444-7.

18. Michael MHT, Wei CJ, Wei LC, et al. Kyphosis correction and height restoration effects of percutaneous vertebroplasty. AJNR Am J Neuroradiol 2003;24:1893-900.

19. McKiernan F, Jensen R, Faciszewski $\mathbf{T}$. The dynamic mobility of vertebral compression fractures. J Bone Min Res 2003;18:24-9.

20. Cotten A, Dewatre F, Cortet B, et al. Percutaneous vertebroplasty for osteolytic metastases and myeloma: affects of the percentage of lesion filling and the leakage of methyl methacrylate at clinical follow-up. Radiology 1996;200:525-30.

21. Weill A, Chiras J, Simon JM, et al. Spinal metastases: indications for and result of percutaneous injection of acrylic surgical cement. Radiology 1996;199:241-7. 